\title{
Performance Analysis of Equalization Techniques for MC-CDMA Systems
}

\author{
Kuzhaloli.S \\ Research Scholar \\ Sathyabama University \\ Chennai, India
}

\author{
Shaji.K.S \\ Principal \\ RIITW \\ Nagercoil, India
}

\begin{abstract}
Nowadays in mobile communication systems, very high bit rates are very essential for transmitting the data and to provide good service for a digital network. This high bit rate leads to Inter symbol Interference (ISI) problems. MC-CDMA technique plays a major role in eliminating these interference problems. In order to compensate this ISI problem, a very effective equalization technique is required. This paper presents the performance analysis of Equalization techniques for an MC-CDMA system which uses BPSK modulation in the presence of Additive white Gaussian Noise (AWGN) over Rayleigh Fading Channel. The analysis is simulated with the help of MATLAB software.
\end{abstract}

\section{Keywords}

MC-CDMA, BPSK, AWGN, Rayleigh fading, MRC, ZF, EGC, MMSE

\section{INTRODUCTION}

MC-CDMA plays a vital role in the wireless indoor communications. Multi Carrier Code Division Multiple Access (MC-CDMA) is a combination of OFDM and CDMA technology. It overcomes the drawbacks of both the technologies. It provides a solution for the efficient utilization of bandwidth, frequency selectivity and diversity and multipath propagation. This paper discusses the equalization techniques to improve Bit Error Rate (BER) and Signal to noise (SNR) Ratio. In Wireless technology, a signal suffering very badly from ISI can be recovered using a efficient equalizer. The various equalizers available and suitable for an MC-CDMA system and their performance is discussed in this paper.

\section{MC-CDMA SYSTEM}

In this MC-CDMA scheme, the spreading sequences are applied in the frequency domain instead of applying them in the time domain. This system can also be viewed as DSCDMA modulated by an OFDM subcarrier [4] [6]. Here, the same symbol is transmitted by different subcarriers. In the MC-CDMA transmitter, the input data is in the serial fashion and this is converted to a parallel data using a serial-toparallel ( $\mathrm{S} / \mathrm{P})$ converter. Then each data is then spread by multiplying each of the users with a unique spreading code. The spreading codes can be an m-sequence, Walsh code, Hadamard codes or Gold codes [2]. The sequences are then converted to time domain samples using a IFFT. They are again converted into a serial data using a Parallel-to-Serial $(\mathrm{P} / \mathrm{S})$ converter and are combined together. This modulated signal is sent to the MC-CDMA receiver. This is signal is equalized and are separated as per their spreading codes. They are then passed through low pass filters and finally multiplexed.

\section{EQUALIZATION TECHNIQUES}

In wireless communication, an equalizer in general is implemented in the receiver side in order to recover the signal very efficiently from the Inter symbol interference problems [5] [7]. This implementation of equalizers improves the Bit Error Rate and hence provides good Signal to Noise ratio [3] [8]. These equalization techniques are also called as combining techniques as the signals from various paths are combined together. The Equalization techniques are as follows:

\subsection{Maximal Ratio Combining (MRC)}

In this MRC technique, each of the signals is multiplied with a weight function which is proportional to the signal amplitude. Hence the diversity branch which has strong signals is amplified further and the branches with weak signals are further attenuated. In this diversity combining technique, signals from various channels are added together and the gain of each channel is proportional to the RMS value of the signal and is inversely proportional to the mean square noise level of that particular channel. This is also called as pre-detection combining or ratio-squared technique and is well suited for independent AWGN channels. The probability of error is given by $\mathrm{Pe}=1 / 2-1 / 2\left(1+1 /\left(\mathrm{E}_{\mathrm{b}} / \mathrm{N}_{0}\right)\right)^{-1 / 2}$.

\subsection{Zero Forcing (ZF) Equalizer}

Zero forcing equalization refers to a technique of linear equalization algorithm. Zero Forcing equalizes by applying the inverse of the channel to the received signal and restores the signal [9]. If the frequency response of the channel is represented by $F(f)$, the zero forcing equalizer $E(f)$ is constructed such that $\mathrm{E}(\mathrm{f})=1 / \mathrm{F}(\mathrm{t})$. Thus a flat frequency response is obtained when the channel and equalizer are combined together and provides a linear phase given by $F(f)$ $\mathrm{E}(\mathrm{f})=1$. This equalizer forces ISI to zero. The probability of error is given by $\mathrm{Pe}=1 / 2\left[1-\left(\left(\mathrm{E}_{\mathrm{b}} / \mathrm{N}_{0}\right) /\left(\left(\mathrm{E}_{\mathrm{b}} / \mathrm{N}_{0}\right)+1\right)\right)^{1 / 2}\right]$.

\subsection{Minimum Mean Square error (MMSE) Equalizer}

Minimum Mean Square Error Equalizer, as it name defines, minimizes the mean square error (MSE). This minimizes the total power of the noise and also the ISI components present in the output [10]. Hence it works well in not only eliminating the interference but also the also components too. This MSE estimator calculates the mean square error for the fitted values of dependant variables. If ' $a$ ' is an unknown variable which depends on a known variable ' $b$ ', an estimator $a^{\wedge}(b)$ is a function of the measurement of ' $b$ ' and hence the mean square error is give by $E\left\{\left[\mathrm{~A}^{\wedge} \mathrm{B}^{2}\right]\right\}$.

\subsection{Equal Gain Combining (EGC)}

In this Equal Gain Combiner, each signal has the same weighted factor without taking its signal amplitude into consideration and all the received signals are added 
coherently. At the receiver, each received path is equalized by compensating with known channel phase. The equalized signals are accumulated and decoded and then the bit errors are counted. It is very simple and easy to implement this equalizer as no channel estimation is required. The probability of error $\mathrm{Pe}=1 / 2\left[1-\left\{\left(\left(\mathrm{E}_{\mathrm{b}} / \mathrm{N}_{0}\right) *\left(\mathrm{E}_{\mathrm{b}} / \mathrm{N}_{0}+2\right)\right)^{1 / 2} /\right.\right.$ $\left.\left.\left(\left(\mathrm{E}_{\mathrm{b}} / \mathrm{N} 0\right)+1\right)\right\}\right]$

\section{SIMULATION RESULTS}

In this paper, performance analysis of equalization techniques for an MC-CDMA system is worked on. Multi Carrier CDMA system combines the benefits of OFDM and CDMA systems. BPSK modulation is technique is used in the presence of Additive White Gaussian Noise (AWGN) in the Rayleigh fading channel [1]. Simulation is done with the help of Matlab software. Number of users considered is two and the number of data sub-carriers is considered to be 16 . Walsh code is used for spreading the sequence. Cyclic prefix is appended for both the users and then they are transmitted into the Rayleigh channel with the addition of Gaussian white noise. The number of tapings for the Rayleigh channel is considered to be four. Finally the cyclic prefix is removed at the receiver side and the equalization of the channel is carried out for all the MRC, ZF, MMSE, EGC combining techniques. The simulated results are compared for the analysis of the performance of each combining technique for an MC-CDMA system. The MMSE equalization and EGC equalization for User $1 \& 2$ is compared in Fig $1 \& 2$ respectively. Fig $3 \& 4$ shows the comparison of $\mathrm{BER} / \mathrm{N}_{0}$ for both MMSE and EGC equalization technique for User 1 \& 2 respectively. Comparison on the performance of all the four equalizers for both User 1 \& 2 is given in Fig 5 \& 6 respectively.

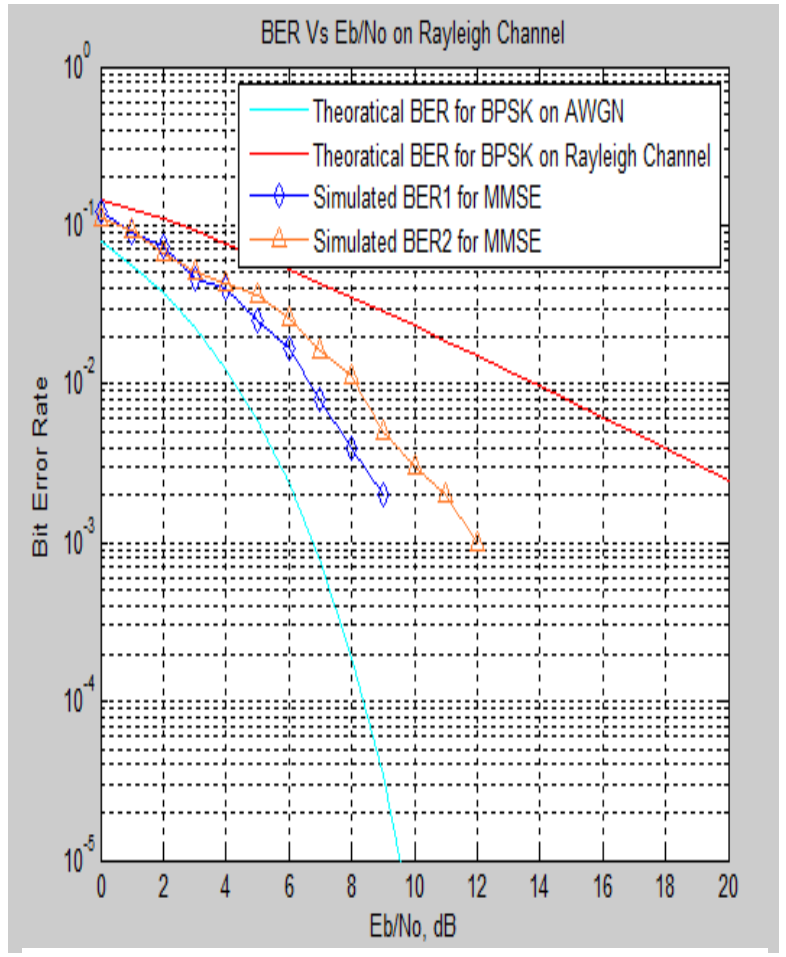

Fig 1: BER/N $\mathrm{N}_{0}$ for MMSE Technique for User 1 \& 2

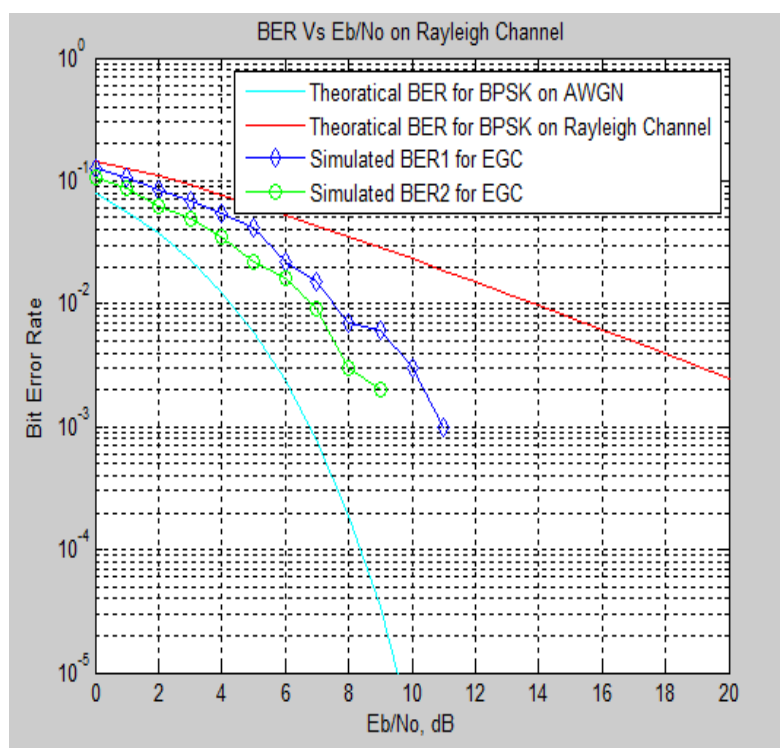

Fig 2: BER/ $\mathbf{N}_{0}$ for EGC Technique for User $1 \& 2$

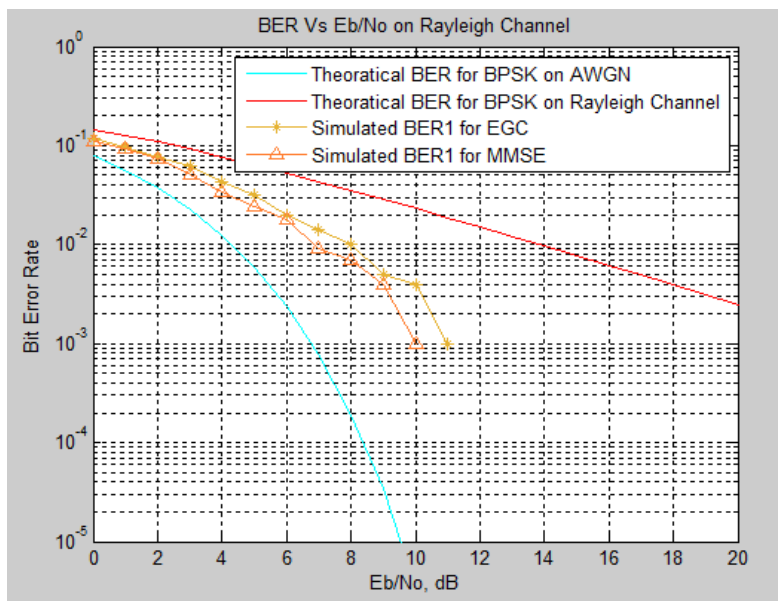

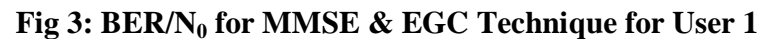

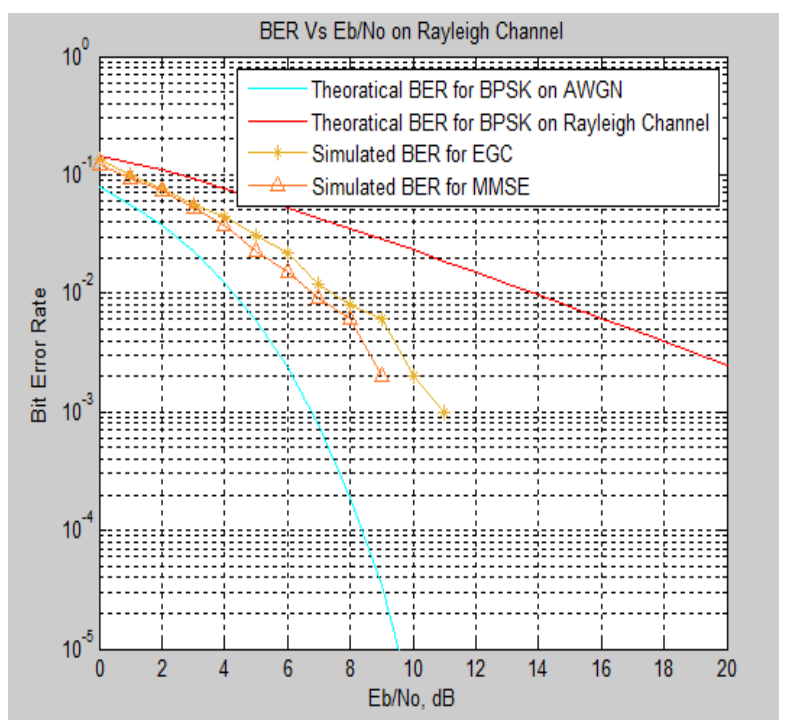

Fig 4: BER/Nn for MMSE \&EGC Technique for User 2 


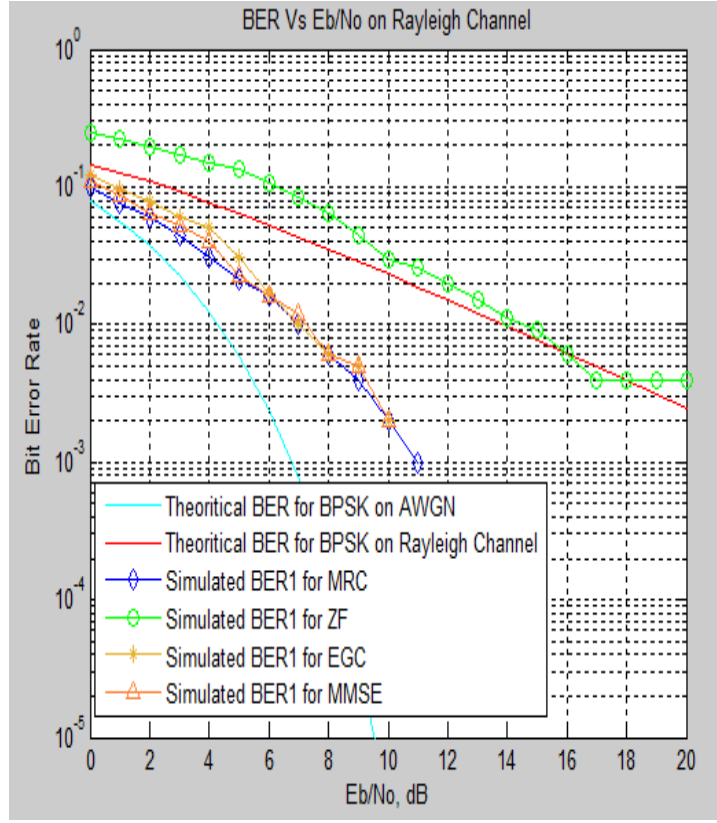

Fig 5: BER/N0 for Equalization Techniques for User 1

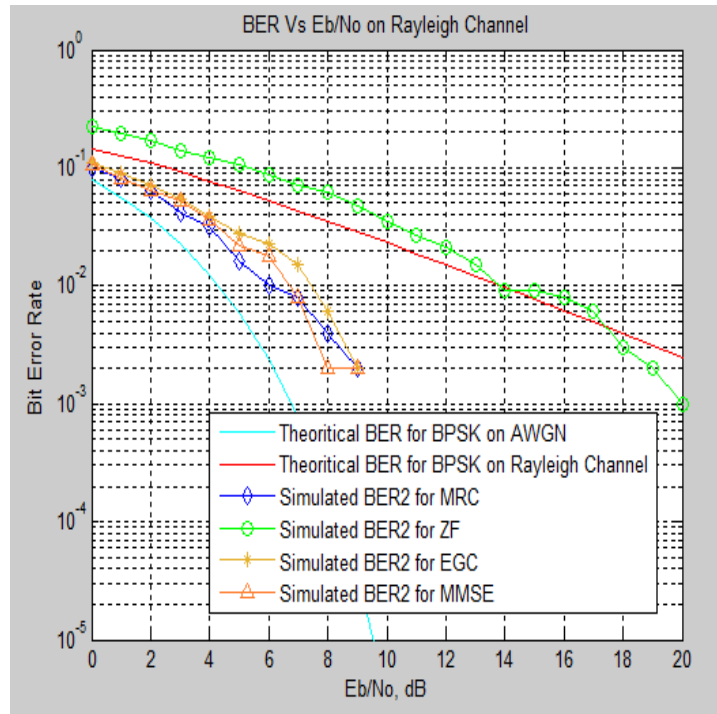

Fig 6: BER/N $\mathrm{N}_{0}$ for Equalization Techniques for User 2

\section{CONCLUSION}

Performance of different equalization techniques has been analyzed to find out the best suitable equalizer for an MCCDMA system. In this paper, four combining techniques namely Maximal Ratio Combining, Zero Forcing, Minimum Mean Square Error and Equal Gain Combining are involved is analyzing the MC-CDMA system which is BPSK modulated in the presence of AWGN in the Rayleigh channel. The simulation parameters are considered and the simulation is carried out using MATLAB. From the results of simulation, the Maximal Ratio Combining (MRC) technique and
Minimum Mean Square Error (MMSE) technique has better performance than Zero Forcing (ZF) and Equal Gain Combining (EGC) Technique in analyzing the Bit Error Rate (BER) of an MC-CDMA system. As an extended future wok, these equalization techniques can be evaluated for different modulation schemes like QPSK, MSK, QAM that are affected by various other fading channels.

\section{REFERENCES}

[1] Satish, V., Suresh, K., \& Swati, B., "Performance Analysis of MCCDMA System in Rayleigh Channel and AWGN Channel Using BPSK Modulation Technique," International Journal of Engineering Research and Applications (IJERA), ISSN:2248-9622, Vol.1, Issue 4, pp.2025-2029.

[2] Manjinder Singh, \& Karamjeet Kaur, (2013 ). "BER Performance of MC-CDMA Using Walsh Code with MSK Modulation on AWGN and Rayleigh Channel," International Journal of Engineering Trends and Technology (IJETT) - Volume 4, Issue 7, PP. No.31663172.

[3] Paramveer Narwal, Sajjan Singh, \& Dr. Prasad, S. V. A. V., (2012 Aug). "Performance Analysis of BER Improvement for Multi Carrie CDMA System," International Journal of Engineering and Innovative Technology (IJEIT), Volume 2, Issue 2, pp.72-74.

[4] Karmjeet Singh \& Er. Rajbir Kaur, (21013 Sep - Oct). "Performance Comparison of Multi-Carrier CDMA Using QPSK and BPSK Modulation," IOSR Journal of Electronics and Communication Engineering (IOSRJECE), Volume 7, Issue 3, pp.61-66.

[5] Jagn Naveen, V., Murali Krishan, K., \& RajaRajeswari, $\mathrm{K}$, (2010 Oct). "Performance analysis of equalization techniques for MIMO systems in wireless communication," International Journal of Smart Home, Vol.4, No.4, PP.no. 47-63.

[6] Ghanim, M. F., \& Abdullah, M. F. L., (2012 Mar). "Multi-user MC-CDMA Using Pseudo Noise Code for Rayleigh and Gaussian Channel,' PIERS Proceedings, Kuala Lumpur, MALAYSIA, pp.882-887.

[7] Dhruv Malik, Deepak Batra, "Comparison of various detection algorithms in a MIMO wireless communication receiver," International Journal of Electronics and Computer Science Engineering (IJECSE), ISSN:22771956, Vol.1, No.3, PP.1678-1685.

[8] Vaishali, W., \& Dr. Chopade, N. B., (2014 May). "Techniques for Improving BER and SNR in MIMO Antenna for Optimum Performance," SSRG International Journal of Electrical and Electronics Engineering (SSRG-IJEEE), ISSN:2348-8379, Vol. 1, Issue. 3, PP.11-14.

[9] Swetamadhab Mahanta, \& Ankit Rajauriam, (2013 April-June) "Analysis of MIMO System Through ZF \& MMSE Detection Scheme," International Journal of Electronics \& Communicational Technology (IJECT)," Vol.4, Issue Spl.4 pp.84-87.

[10] Tamilarasan, N., Nithyanandan, L., (2014 Jan) "Performance of MIMO MC-CDMA System with Channel Estimation and MMSE Equalization," Journal of Theoretical and Applied Information Technology (JATIT), Vol.59, No.2, pp.436-441. 